\title{
LAS FLORES DE MYRTILLOCACTUS GEOMETRIZANS (CACTACEAE): MORFOLOGÍA Y ARTRÓPODOS VISITADORES EN UN GRADIENTE GEOGRÁFICO
}

\section{THE FloWers of MYRTILlocactus geOMETRIZANS (CACTACEAE): MORPHOLOGY AND ARTHROPOD VISITORS IN A GEOGRAPHICAL GRADIENT}

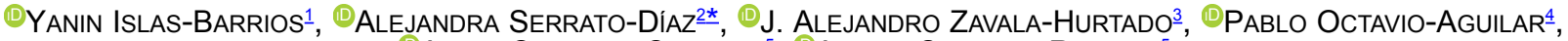

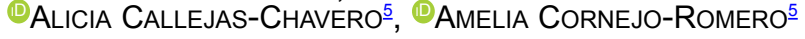

'Doctorado en Ciencias Biológicas y de la Salud, División de Ciencias Biológicas y de la Salud, Universidad Autónoma Metropolitana, Ciudad de México, México

¿Universidad Autónoma Metropolitana Iztapalapa, Departamento de Hidrobiología, División de Ciencias Biológicas y de la Salud, Colonia Vicentina, México.

${ }^{3}$ Universidad Autónoma Metropolitana Iztapalapa, Departamento de Biología, División de Ciencias Biológicas y de la Salud, Colonia Vicentina, México.

${ }^{4}$ Universidad Autónoma del Estado de Hidalgo, Laboratorio de Genética, Centro de Investigaciones Biológicas, Hidalgo México.

${ }^{5}$ Laboratorio de Ecología Vegetal, Departamento de Botánica de la Escuela Nacional de Ciencias Biológicas, IPN. Ciudad de México, México.

*Autor para correspondencia: alej@xanum.uam.mx

\section{Resumen}

Antecedentes: Las flores son elementos reproductivos costosos que presentan balances costo/beneficio en sus estructuras. La diversidad de éstas se ha atribuido a factores bióticos como las interacciones con sus visitadores florales y abióticos como las características del ambiente.

Preguntas: ¿La morfología floral de M. geometrizans está relacionada con los tipos de vegetación? ¿La comunidad de artrópodos visitadores florales se relaciona con la morfología floral y/o el tipo de vegetación? ¿La morfología floral y la comunidad de artrópodos tendrán relación con la perturbación del ambiente?

Especie de estudio: Myrtillocactus geometrizans (Mart. ex Pfeiff.) Console

Sitio de estudio y fecha: Selva Baja Caducifolia y Matorral Xerófilo en las Reservas de la Biosfera: Tehuacán-Cuicatlán, Puebla y Barranca de Metztitlán, Hidalgo. México. Marzo 2017.

Métodos: En los cuatro sitios (dos días por sitio), durante el pico de floración de M. geometrizans, se calculó el índice de disturbio, se recolectaron 30 flores $(\mathrm{N}=120)$ y los artrópodos visitadores florales. Se determinaron la morfología floral y los artrópodos.

Resultados: Los sitios de la Barranca de Metztitlán presentaron el mayor índice de disturbio, las flores más chicas y mayor número de estructuras florales. Los visitadores florales fueron más abundantes en los matorrales xerófilos, particularmente en el sitio más perturbado donde el grupo funcional más relevante fue el de los polinizadores.

Conclusiones: Se encontraron diferencias significativas en la morfología floral de M. geometrizans relacionadas a los tipos de vegetación y ubicación geográfica. Se reportó una compensación entre tamaño y producción de estructuras florales.

Palabras clave: Disturbio antrópico, garambullo, grupos funcionales, matorral xerófilo, selva baja caducifolia.

\begin{abstract}
Background: Flowers are expensive reproductive elements that have cost/benefit balances in their characters. The diversity of the floral structures has been attributed to biotic factors such as the interactions with their floral visitors and abiotic factors such as the characteristics of the environment.

Question: Is there a relationship between the floral morphology of $M$. geometrizans and the vegetation type? Is the community of arthropod floral visitors associated with floral morphology and/or the vegetation type? Will the floral morphology and the community of arthropod floral visitors be associated with the disturbance level of the environment?

Studied species: Myrtillocactus geometrizans (Mart. ex Pfeiff.) Console

Study site and dates: Tropical deciduous forest and xerophilous scrub in the Tehuacán-Cuicatlán Biosphere Reserve, Puebla, and in the Barranca de Metztitlán Biosphere Reserve, Hidalgo, Mexico. March 2017.

Methods: At the four sites (two days per site), during the flowering peak of $M$. geometrizans, the disturbance index was calculated, 30 flowers $(\mathrm{N}=120)$ and the arthropods floral visitors were collected. Floral morphology and arthropods were determined.

Results: Barranca de Metztitlán sites showed the highest disturbance index, the smallest flowers, and the highest production of floral structures. Flower visitors were more abundant in xerophilous scrubs, particularly in the most disturbed site, where the most relevant functional group was the pollinators.

Conclusions: Significant differences were found in the floral morphology of M. geometrizans related to vegetation types and geographic location. A trade-off between the size and production of floral characters was reported.
\end{abstract}

Keywords: Anthropogenic disturbance, garambullo, functional groups, tropical deciduous forest, xerophilous scrub. 
En México, cerca del $60 \%$ del territorio está compuesto por zonas áridas y semiáridas con una alta diversidad biológica y endemismos (Rzedowski 2006). Estas regiones han sido centro de diversificación de muchos grupos, entre los que se encuentran las cactáceas (Bravo-Hollis \& SánchezMejorada 1991). Actualmente, en México, se tiene un registro de alrededor de 52 géneros y 850 especies de cactáceas, de las cuales aproximadamente el $84 \%$ son endémicas (Bravo-Hollis \& Sánchez-Mejorada 1991, Arias et al. 2005). Este grupo de plantas presenta características morfológicas y fisiológicas que responden a la baja disponibilidad de agua, lo que le ha permitido ser exitoso en estos ambientes (Bravo-Hollis \& Sánchez-Mejorada 1991).

Dentro de las cactáceas endémicas de México, se encuentra Myrtillocactus geometrizans (Mart. ex Pfeiff.) Console, 1897, comúnmente conocida como garambullo. Esta especie resulta particularmente interesante porque, a diferencia de la mayoría de las cactáceas endémicas, presenta una amplia distribución en el país (Casas et al. 1999). Presenta un periodo de floración (febrero a abril) que coincide con el de pocas especies, lo que la hace ser uno de los pocos recursos disponibles para un gran número de artrópodos visitadores florales (Arias et al. 1997, RosasGarcía 2010, Maqueda-Díaz \& Callejas-Chavero 2018).

Las flores son elementos reproductivos costosos, que presentan balances costo/beneficio entre sus estructuras, por ejemplo, el tamaño y cantidad de elementos de perianto, de estambres y el estigma, con la finalidad de favorecer la reproducción (Harder \& Barrett 2006). Existen numerosos estudios que demuestran que las plantas invierten en flores con elementos de perianto grandes y llamativos o en un incremento en el número de estambres o polen, para atraer a los polinizadores (Armbruster 1997, Harder \& Barrett 2006). La morfología de las flores es en gran medida el resultado de la presión selectiva ejercida por los polinizadores para beneficiar las estructuras florales que favorecen la exportación y recepción del polen (Mandujano et al. 2010, Barrett 2010, Woźniak \& Sicard 2018). Incluso en plantas hermafroditas, que pudieran no necesitar de polinizadores, se han desarrollado sistemas para favorecer la exportación de polen (Mandujano et al. 2010). Por ejemplo, ciertos acomodos espaciales de anteras y estigmas, conocidos como hercogamia, evitan la autopolinización (Ganders 1979, Mandujano et al. 2010). Así, las flores con estilo largo y estambres cortos (morfo Pin) reciben polen; mientras que flores con estilo corto y estambres largos (morfo Thrum) lo donan (Ganders 1979, Mandujano et al. 2010).

La diversidad de estructuras florales también está relacionada a otros factores bióticos, como lo son los visitadores florales no polinizadores, entre los que se encuentran artrópodos depredadores y parasitoides de los polinizadores, robadores de néctar y de polen (Geber \& Moeller 2006, Maqueda-Díaz \& Callejas-Chavero 2018).
También, se ha documentado variación en la morfología floral relacionada a condiciones ambientales que enfrentan las poblaciones como temperatura y radiación solar (Aguilar-García et al. 2018) y eventos como el disturbio que pueden influir sobre las estructuras florales, al cambiar las condiciones físicas del ambiente, modificar la composición de las comunidades, la diversidad y las relaciones interespecíficas (Strauss \& Whittall 2006, Aizen 2007, Krishnadas et al. 2018). Estudios intrapoblacionales realizados sobre las flores de $M$. geometrizans han mostrado variaciones morfológicas en respuesta a microambientes (Aguilar-García et al. 2018), además de una diversa comunidad de artrópodos visitadores florales (Rosas-García 2010, Maqueda-Díaz \& Callejas-Chavero 2018). Sin embargo, no se ha estudiado si existe variación en la morfología floral de esta especie en diferentes tipos de vegetación, ni bajo diferentes grados de perturbación antrópica y si estos factores influyen en la composición de los visitadores florales.

En el presente trabajo se estudió, durante el pico de floración, la morfología floral de $M$. geometrizans en dos tipos de vegetación (selva baja y matorral xerófilo) de las Reservas de la Biosfera de Tehuacán-Cuicatlán y Barranca de Metztitlán, México. Se analizó si la comunidad de artrópodos visitadores florales responde a la morfología floral y si el índice de disturbio antrópico influye en este sistema. Se plantearon las siguientes hipótesis: 1) Debido a que enfrentan presiones de selección parecidas, las flores tendrán mayor similitud morfológica relacionada al tipo de vegetación, independiente a la cercanía geográfica de los sitios; 2) Las flores grandes, por ser más atractivas, tendrán una comunidad de artrópodos con mayor riqueza y abundancia; 3) La perturbación genera nuevas condiciones microambientales que pueden favorecer el establecimiento de especies; como consecuencia, se espera que los sitios más perturbados presenten mayor abundancia de artrópodos visitadores florales.

\section{Material y métodos}

Área de estudio. Este trabajo se llevó a cabo en dos Reservas de la Biósfera en las que se distribuye M. geometrizans. En la Reserva de la Biósfera de la Barranca de Metztitlán (RBBM), Hidalgo, que se caracteriza por tener clima semiárido (CONANP 2003), con una precipitación media anual $427 \mathrm{~mm}$ y temperatura media anual de $22{ }^{\circ} \mathrm{C}$ (SEMARNAP 1999); se ubicaron los sitios uno y dos. El primer sitio se ubicó en matorral xerófilo (1MMX) (20²6' 48.3" N 98 40' 30.72" O, 1,611 m) y el segundo en selva baja caducifolia (2MSB) $\left(20^{\circ} 28^{\prime} 33.492^{\prime \prime}\right.$ N $98^{\circ} 40^{\prime} 38.46^{\prime \prime} \mathrm{O}, 1,316 \mathrm{~m}$ ). En la Reserva de la Biósfera Tehuacán-Cuicatlán (RBTC), Oaxaca, que también se caracteriza por su clima semiárido (Valverde et al. 2007), con una precipitación media anual de $380 \mathrm{~mm} \mathrm{y}$ 
temperatura media anual de $21{ }^{\circ} \mathrm{C}$ (Arias et al. 1997, Rzedowski 2006); se ubicaron los sitios tres y cuatro. El tercer sitio se localizó en matorral xerófilo (3TMX) (18 ${ }^{\circ} 19^{\prime}$ $\left.43.68^{\prime \prime} \mathrm{N} 97^{\circ} 27^{\prime} 9.50^{\prime \prime} \mathrm{O}, 1,438 \mathrm{~m}\right)$ y el cuarto en selva baja

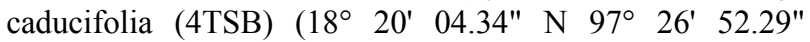
O, 1,709 m) (Figura. 1).

Sistema de estudio. Myrtillocactus geometrizans (Mart. ex Pfeiff.) Console es un cactus arborescente (2-5 m de altura), endémico y ampliamente distribuido en todo México (Bravo-Hollis \& Sánchez-Mejorada 1978). Su tallo principal es corto y ramificado (Arias et al. 1997). Cada rama tiene entre cinco y siete costillas (Arias et al. 1997). Presenta entre 4 a 9 espinas radiales de 0.2 a $5.0 \mathrm{~cm}$. En la areola se pueden desarrollar de una a varias flores (Arias et al. 1997). Tiene flores hermafroditas, blancas con tintes purpúreos, con antesis diurna $(\approx 07: 00$ a 14:00 h), de un solo día, de 2-3 cm de largo y $2.5-3.5 \mathrm{~cm}$ de ancho (Arias et al. 1997, Rosas-García 2010). La especie florece de febrero a abril, y se ha documentado que sus principales polinizadores son abejas (Arias et al. 1997, Rosas-García 2010, Maqueda-Díaz \& Callejas-Chavero 2018).
Caracterización del ambiente. En cada sitio se realizó un análisis de perturbación siguiendo el procedimiento que Martorell \& Peters (2009) desarrollaron para evaluar ambientes áridos. Este método permite el cálculo de un índice de disturbio (ID) a partir de la estimación de 13 parámetros, divididos en tres categorías: actividades humanas $(\mathrm{AH})$, ganadería $(\mathrm{G})$ y deterioro del hábitat $(\mathrm{DH})$. Estos parámetros se registraron en tres transectos de $50 \mathrm{~m}$ de largo por $1 \mathrm{~m}$ de ancho para cada uno de los sitios de estudio.

Morfología floral. Se evaluó la morfología floral durante el pico de floración (comunicación personal con la gente local) con la finalidad de contar con un mayor número de flores. En los sitios 1MMX y 2MSB se realizó el 25 y 26 de marzo, mientras que para los sitios 3TMX y 4TSB los días 11 y 12 de marzo del año 2017. En cada sitio se seleccionaron al menos siete garambullos. A una altura máxima de 2 metros (altura promedio de los individuos reproductivos más pequeños), se recolectaron de 3 a 5 flores por individuo, considerando todos los sectores acimutales (30 flores por sitio) para caracterizar el promedio de las
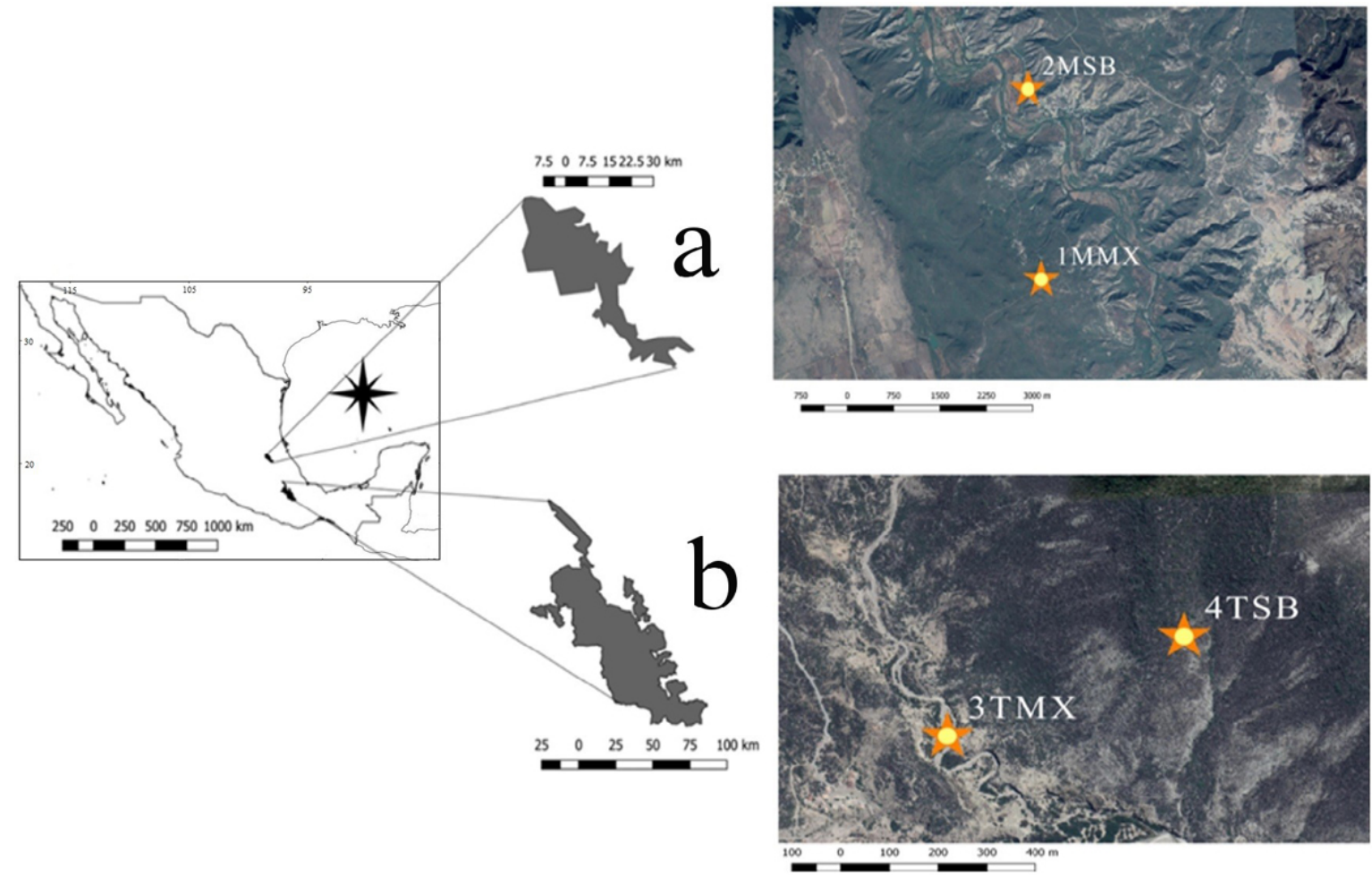

Figura 1. Ubicación de los sitios de estudio, a) Reserva de la Biósfera Barranca de Metztitlán donde se encuentran los sitios 1MMX, Matorral Xerófilo de Metztitlán; y 2MSB, Selva Baja de Metztitlán; y b) Reserva de la Biósfera Tehuacán-Cuicatlán donde se encuentran los sitios 3TMX, Matorral Xerófilo de Tehuacán y 4TSB, Selva Baja de Tehuacán. 
flores, sin considerar diferencias intraindividuales. Para registrar el diámetro de las 120 flores durante la antesis (Dmt Ttl), a cada una se le tomó una fotografía in situ, de frente, montada en una base con referencia métrica para que todas estuvieran en un mismo plano (porque al ser almacenadas se pierde el máximo de apertura floral) (Martínez-Ramos et al. 2017). Posteriormente, las flores se almacenaron en tubos de $50 \mathrm{ml}$ con glicerina:alcohol 50:50 (Páez-Gerardo et al. 2005). En el laboratorio se registraron las características morfométricas de las estructuras florales. Se tomaron medidas de diámetro de la flor (Dmt Ttl) en las imágenes tomadas in situ mediante el software ImageJ 1.5 (Rasband 1997). Con un vernier digital, de $0.01 \mathrm{~mm}$ de resolución (Mitutuyo, Japón), se midió altura total de la flor (Alt Ttl), diámetro del tubo floral (Dmt Tb Flr), diámetro ecuatorial del ovario (Dmt Ectr Ovr), diámetro polar del ovario (Dmt Pl Ovr), longitud de estambres (Lng Estb), longitud del estigma (Lng Estg) y longitud de lóbulos (Lng Lbs). Con ayuda de un microscopio estereoscópico Leica EZ4, se contabilizó el número de estambres (No Estb), de lóbulos (No Lbs), de elementos del perianto (E Prnt) y de óvulos (No Ovl). Además, para la determinación del tipo de hercogamia (Hrc) se midió la longitud del estilo más el estigma y de los estambres (Figura 2).

Artrópodos visitadores de las flores. Durante los días de recolecta, en cada sitio se seleccionaron al azar tres individuos de garambullo. Se recolectaron todos los artrópodos visitadores de las flores con aspiradores, redes entomológicas, pinceles y recolecta directa. La recolecta se realizó durante dos días en cada sitio, de 7:00 a 14:00 h, de manera continua durante las 7 horas, por 5 recolectores en cada sitio ( $60 \mathrm{~h}$ de recolecta por sitio). Los ejemplares recolectados se conservaron en alcohol etílico al $86 \%$
(Morinière et al. 2017). Los artrópodos se separaron por morfoespecie (Msp) con ayuda de un microscopio estereoscópico Leica EZ4 y se identificaron con claves taxonómicas (Triplehorn \& Johnson 2005) y con la ayuda del especialista Jorge Orendain-Méndez de la Universidad Autónoma Metropolitana-Iztapalapa. Se revisó bibliográficamente el grupo funcional al que pertenecen las Msp identificadas (Toft \& Kimsey 1982, Morón \& Terrón 1988, Borror et al. 1989, Cardel et al. 1997, Terrón et al. 1998, Moya-Raygoza et al. 2004, Ríos-Casanova et al. 2004, Strauss 2012, Martínez-Hernández \& CallejasChavero 2015, Luna de la Torre et al. 2016, Maqueda-Díaz \& Callejas-Chavero 2018, Navarrete-Jiménez 2018).

Análisis estadísticos. Para estimar el ID se elaboró una matriz con los datos de los 13 parámetros obtenidos en el análisis de perturbación y se aplicó un análisis de componentes principales (ACP) centrado, siguiendo el procedimiento propuesto por Martorell \& Peters (2009), en el paquete estadístico MVSP 3.13 (Kovach 2004). Como el ACP centrado genera valores negativos y positivos, el primer eje se re-escaló para obtener un ID que abarcara un rango de 0 a 100 , donde el 0 corresponde al transecto que presentó el menor disturbio antrópico y el 100 al que presentó el mayor. Se promedió el valor de ID para los tres transectos de cada sitio. Dado que los puntajes del ACP para cada sitio integran una combinación lineal de los valores registrados para cada variable, se desglosó la contribución al ID de cada categoría de disturbio (AH, G y $\mathrm{DH})$ al sumar los términos lineales correspondientes a cada una de ellas (Martorell \& Peters 2005).

Para identificar si había diferencias entre las flores de los cuatro sitios en función de las 14 estructuras florales, se realizó un análisis discriminante generalizado con el
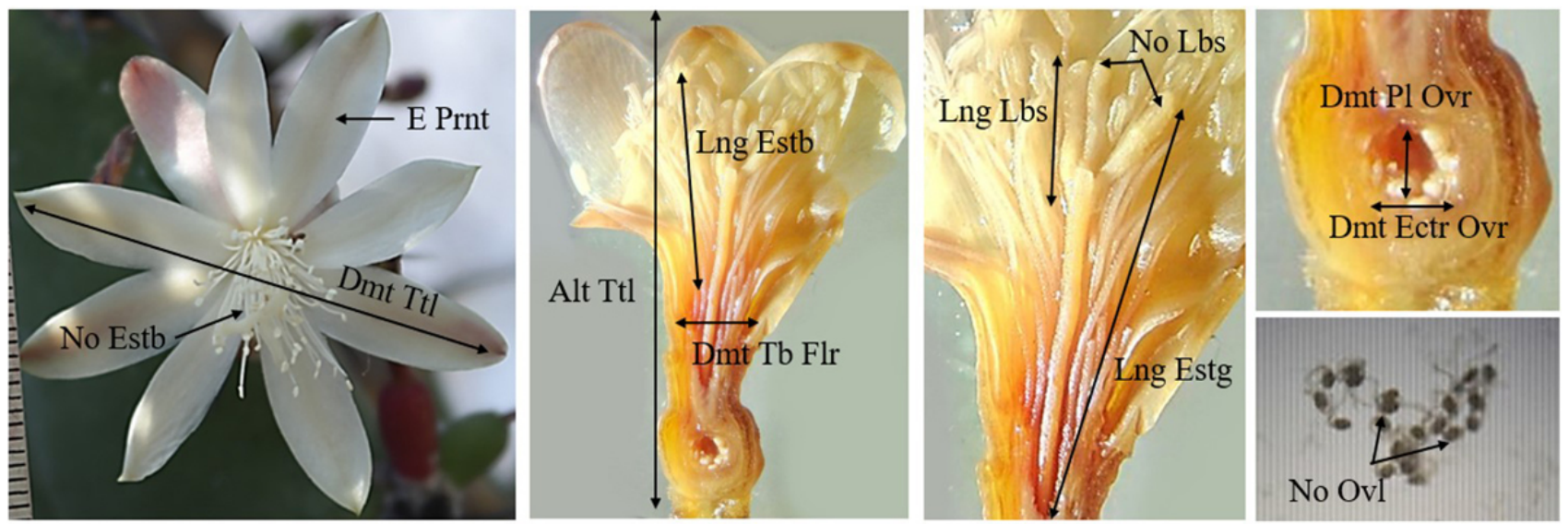

Figura 2. Estructuras florales usadas para el análisis de la morfología floral: número de estambres (No Estb), elementos del perianto (E Prnt), número de óvulos (No Ovl) y número de lóbulos (No Lbs). Se tomaron medidas del diámetro total durante la antesis (Dmt Ttl), longitud de lóbulos (Lng Lbs), longitud de estigma (Lng Estg), longitud de estambres (Lng Estb), diámetro de tubo floral (Dmt Tb Flr), diámetro polar del ovario (Dmt Pl Ovr), diámetro ecuatorial ovario (Dmt Ectr Ovr) y altura total de la flor (Alt Ttl). 
software SPSS Base Statistics v.19 (Norusis 2011). Para comparar las estructuras florales de los cuatro sitios, se utilizaron pruebas no paramétricas de Kruskal-Wallis. En los casos en que se detectaron diferencias significativas, se realizaron comparaciones pareadas con pruebas de $U$ de Mann-Whitney, estimando los valores de $p$ con la corrección de Bonferroni en el paquete estadístico PAST (Hammer et al. 2001). Se analizó la normalidad de los datos de la longitud del estilo más el estigma y la longitud de los estambres con la prueba de Kolmogorov-Smirnov y la homogeneidad de las variaciones con la prueba de Levene en el software SPSS Base Statistics v.19 (Norusis 2011). Para determinar la hercogamia se comparó, con una prueba de $t$-Student pareada, la longitud del estilo más el estigma con la de los estambres en el paquete estadístico PAST (Hammer et al. 2001).

Para evaluar la similitud entre las comunidades de artrópodos de los cuatro sitios se realizó un agrupamiento con el método de Bray-Curtis con el algoritmo UPGMA, utilizando un procedimiento de remuestreo con 1,000 repeticiones bootstrap para tener el valor de soporte de cada rama (Sneath \& Sokal 1973), en el paquete estadístico PAST (Hammer et al. 2001). Para analizar la correlación entre la comunidad de artrópodos y las estructuras florales se realizó un análisis canónico de correspondencia (ACC). Finalmente, se estimó la correlación entre la comunidad de artrópodos y el índice de disturbio de los sitios, con un ACC. Estos análisis se realizaron con el paquete MVSP 3.13 (Kovach 2004).

\section{Resultados}

Caracterización del ambiente. El primer y segundo eje del análisis de componentes principales explicaron el $43.08 \mathrm{y}$ $27.02 \%$ de la varianza total. Este análisis mostró un gradiente a lo largo del cual se ordenaron los cuatro sitios estudiados. Se encontraron diferencias muy marcadas en los valores del índice de disturbio (ID) entre las reservas de Metztitlán y de Tehuacán ( $\underline{\text { Tabla } 1}$ ). El valor más alto de ID se presentó en el sitio $1 \mathrm{MMX}$ (ID = 79.79), donde la ganadería fue el principal agente de disturbio, seguido por el sitio 2MSB (ID = 33.39), donde el principal agente de disturbio fue el de las actividades humanas. El sitio de 4TSB presentó un ID casi cinco veces menor (ID = 8.23), con una contribución similar de actividades humanas y ganadería. Finalmente, el sitio menos perturbado fue el 3TMX con un ID de apenas 2.78, donde todos sus componentes presentaron valores similares ( $\underline{\text { Tabla } 1)}$.

Morfología floral. El análisis discriminante generalizado reveló que existen diferencias significativas entre las estructuras de las flores de $M$. geometrizans entre los cuatro sitios (Lambda de Wilks $=0.08 ; p<0.001)$ ( $\underline{\text { Figura 3 }}$ ). La matriz de estructura, integrada por los valores de correlación entre las variables y las funciones discriminantes, indicó que las estructuras florales que más contribuyeron a las diferencias entre sitios en la función 1 fueron: diámetro total de la flor (-0.522), número de óvulos (0.322) y longitud de lóbulos (-0.205). Mientras que en la función 2 fueron: diámetro ecuatorial del ovario (0.609), longitud de estigma (0.381) y diámetro del tubo floral (0.272).

Tabla 1. Índice de disturbio re-escalado (de 0 a 100) y su desglose en sus tres componentes para los cuatro sitios ubicados en las Reservas de la Biosfera de Metztitlán (1MMX = Matorral Xerófilo; $2 \mathrm{MSB}=$ Selva Baja) y Tehuacán (3TMX = Matorral Xerófilo; 4TSB $=$ Selva Baja). ID = Índice de Disturbio, $\mathrm{AH}=$ Actividades Humanas, $\mathrm{G}=$ Ganadería y $\mathrm{DH}=$ Deterioro del Hábitat.

\begin{tabular}{ccccc}
\hline & AH & G & DH & ID \\
\hline 1MMX & 27.59 & 31.82 & 20.37 & 79.79 \\
2MSB & 12.89 & 8.51 & 11.99 & 33.39 \\
3TMX & 0.80 & 0.94 & 1.03 & 2.78 \\
4TSB & 2.27 & 2.56 & 3.40 & 8.23 \\
\hline
\end{tabular}

En la Reserva de la Barranca de Metztitlán se encontraron flores con los valores más bajos en altura total, longitud de estambres y longitud de lóbulos. Pero con mayor número de óvulos, estambres y elementos del perianto (Tabla 2). En la Reserva de Tehuacán-Cuicatlán las flores mostraron la mayor altura total de la flor, de lóbulos y estambres. Pero, menor número de óvulos, estambres y elementos del perianto (Tabla 2).

De acuerdo con la prueba de $t$-Student pareada, las flores de los sitios de matorral xerófilo presentaron hercogamia (1MMX $t=-3.46, p=0.001$ y 3TMX $t=-2.68, p=0.011$ ). En el $63 \%$ de los casos los estambres fueron más largos que el estilo más estigma (morfo Thrum). Mientras que, en la selva baja, las flores no presentaron hercogamia a pesar de que predominó el morfo del estigma más estilo más largo que los estambres (2MSB $t=1.81, p=0.081$ y 4TSB $t=1.16, p=0.25$ ) (morfo Pin) (Tabla 2).

Artrópodos visitadores de las flores. Se registraron 329 individuos de artrópodos visitadores de las flores de M. geometrizans. En el sitio $1 \mathrm{MMX}$ se recolectaron 126 individuos; en el 2MSB, 63; en el 3TMX, 81 y en el 4TSB, 59. Se separaron en $106 \mathrm{Msp}$ pertenecientes a 5 órdenes, Hymenoptera $(\mathrm{N}=259)$, Diptera $(\mathrm{N}=40)$, Coleoptera $(\mathrm{N}=22)$, Thysanoptera $(\mathrm{N}=1)$ y Araneae $(\mathrm{N}=7)$ (Apéndice 1). 


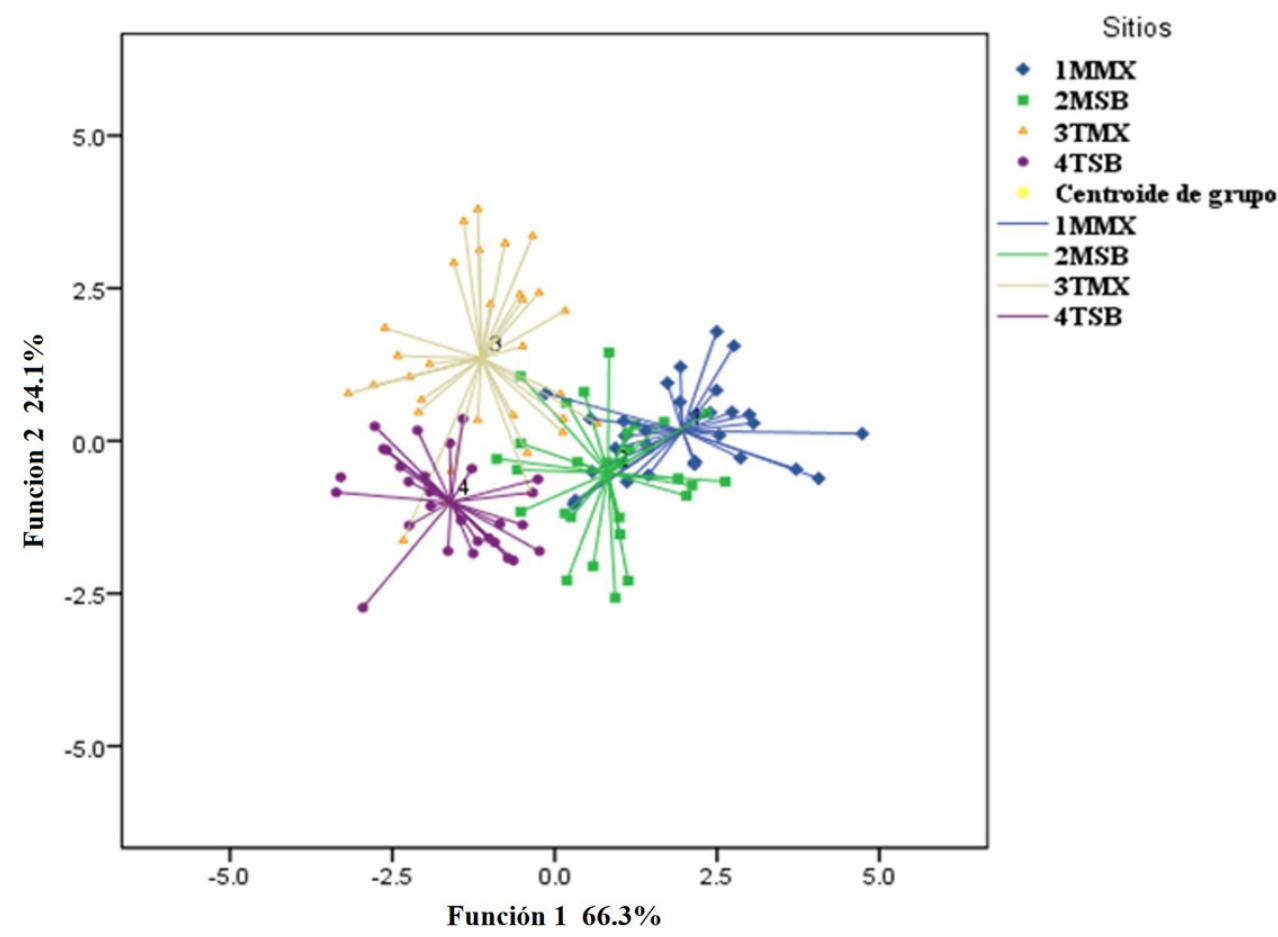

Figura 3. Diagrama de dispersión generado por análisis discriminante generalizado, que indicó diferencias significativas de las flores entre sitios. 1MMX, Matorral Xerófilo de Metztitlán; 2MSB, Selva Baja de Metztitlán; 3TMX, Matorral Xerófilo de Tehuacán; 4TSB, Selva Baja de Tehuacán.

El orden con mayor número de familias fue Hymenoptera con ocho, seguido por Diptera (5), Coleoptera (3) y Araneae (2) (Apéndice 1). La familia con mayor número de individuos recolectados fue Formicidae con 130, seguida de Apidae con 48 y Vespidae con 37 (Apéndice 1). Estas familias también presentaron el mayor número de $M s p$, Formicidae con 30, Apidae con 21 y Vespidae con 14.

Se identificaron bibliográficamente nueve grupos funcionales de artrópodos, el más representativo fue el de los polinizadores con el $56.1 \%$ de la recolecta, pertenecientes a las familias Anthophoridae, Apidae, Halictidae, Syrphidae y Vespidae. El segundo grupo funcional más abundante fue el de los depredadoresnectarívoros con el $10.4 \%$, registrados en la familia Formicidae; seguidos de los nectarívoros con el $8.5 \%$, donde se identificaron las familias Formicidae y Thripidae (Apéndice 1).

El análisis de agrupamiento de las comunidades de artrópodos de los cuatro sitios indicó que la composición no depende del tipo de vegetación ni de la separación geográfica. Este análisis separó al sitio $1 \mathrm{MMX}$ del resto (correlación cofenética de 0.85), el cual se caracterizó por ser el más perturbado y presentar mayor abundancia de artrópodos (Figura 4).

Los resultados de los análisis de correspondencia explicaron en un $95.1 \%$ que los visitadores florales presentaron mayor abundancia en el sitio 1MMX que se caracteriza por tener el mayor ID (Figura 5 A). Por otro lado, indicaron en un $97.52 \%$ que los órdenes de visitadores florales se correlacionan únicamente con la hercogamia y no con el resto de las estructuras florales (Figura 5 B).

\section{Discusión}

Los resultados de este estudio indican que los sitios de la Barranca de Metztitlán presentan la mayor perturbación antrópica. En contraste, los sitios de la Reserva de Tehuacán-Cuicatlán registraron valores de perturbación muy bajos. A pesar de ser una Reserva de la Biósfera, en Metztitlán se han reportado áreas de cultivo (principalmente maíz, frijol, calabaza, chile, tomate verde, jitomate y agave) e ingreso de ganado (cabras, ovejas y vacas) (Moreno et al. 2007) que contribuyen al disturbio de los sitios. En contraste, los sitios de estudio de Tehuacán se encuentran dentro del área de conservación del Jardín Botánico "Helia Bravo Hollis" donde no hay ingreso de ganado, ni zonas de cultivo. Por lo que, en los sitios de la Reserva de Metztitlán, las especies, incluyendo a $M$. geometrizans, enfrentan presiones de selección como mayor riesgo de desecación, mayor radiación solar e incremento del viento (Krishnadas et al. 2018, Stone et al. 2018). 
Tabla 2. Estructuras florales de M. geometrizans (media \pm EE) en cuatro sitios ubicados en las Reservas de la Biosfera de Metztitlán (1MMX, Matorral Xerófilo; 2MSB, Selva Baja) y Tehuacán (3TMX, Matorral Xerófilo; 4TSB, Selva Baja). En negritas se presentan los valores más altos. Letras diferentes indican diferencias significativas.

\begin{tabular}{|c|c|c|c|c|}
\hline Estructuras florales & $\begin{array}{c}1 \mathrm{MMX} \\
\text { Media } \pm \text { EE }\end{array}$ & $\begin{array}{c}2 \mathrm{MSB} \\
\text { Media } \pm \text { EE }\end{array}$ & $\begin{array}{c}\text { 3TMX } \\
\text { Media } \pm \text { EE }\end{array}$ & $\begin{array}{c}\text { 4TSB } \\
\text { Media } \pm \text { EE }\end{array}$ \\
\hline Altura total $(\mathrm{cm})$ & $2.49^{\mathrm{a}} \pm 0.041$ & $2.65^{\mathrm{a}} \pm 0.067$ & $2.92^{b} \pm 0.054$ & $3.04^{b} \pm 0.036$ \\
\hline Diámetro total $(\mathrm{cm})$ & $3.34^{\mathrm{a}} \pm 0.63$ & $\mathbf{3 . 7 0}^{\mathrm{b}} \pm 0.092$ & $3.50^{\mathrm{bc}} \pm 0.065$ & $3.41^{\text {ac }} \pm 0.044$ \\
\hline Diámetro del tubo floral $(\mathrm{cm})$ & $3.34^{\mathrm{a}} \pm 0.014$ & $0.34^{\mathrm{a}} \pm 0.016$ & $0.34^{\mathrm{a}} \pm 0.014$ & $\mathbf{0 . 4 1}^{\mathrm{b}} \pm 0.014$ \\
\hline Diámetro ecuatorial del ovario $(\mathrm{cm})$ & $0.22^{\mathrm{ac}} \pm 0.009$ & $0.20^{\mathrm{ab}} \pm 0.011$ & $\mathbf{0 . 2 9}{ }^{c} \pm 0.022$ & $0.18^{b} \pm 0.007$ \\
\hline Diámetro polar del ovario $(\mathrm{cm})$ & $0.30^{\mathrm{a}} \pm 0.012$ & $0.31^{\mathrm{a}} \pm 0.012$ & $0.32^{\mathrm{a}} \pm 0.036$ & $\mathbf{0 . 3 3}^{\mathrm{a}} \pm 0.013$ \\
\hline Longitud de estambres $(\mathrm{cm})$ & $1.23^{\mathrm{a}} \pm 0.020$ & $1.21^{\mathrm{ab}} \pm 0.042$ & $1.27^{\mathrm{ab}} \pm 0.033$ & $1.32^{\mathrm{b}} \pm 0.024$ \\
\hline Longitud de estigma $(\mathrm{cm})$ & $1.17^{\mathrm{ab}} \pm 0.022$ & $1.26^{\mathrm{bc}} \pm 0.043$ & $1.16^{\mathrm{b}} \pm 0.045$ & $\mathbf{1 . 3 6}^{\mathrm{c}} \pm 0.036$ \\
\hline Longitud de lóbulos (cm) & $0.47^{\mathrm{a}} \pm 0.017$ & $0.46^{\mathrm{ab}} \pm 0.026$ & $0.54^{\mathrm{ab}} \pm 0.029$ & $\mathbf{0 . 5 6}^{\mathrm{b}} \pm 0.020$ \\
\hline Número de estambres & $\mathbf{9 2 . 6 0} 0^{\mathrm{a}} \pm 3.93$ & $80.37^{\mathrm{a}} \pm 3.34$ & $89.17^{\mathrm{a}} \pm 2.32$ & $86.90^{\mathrm{a}} \pm 2.13$ \\
\hline Número de lóbulos & $4.67^{\mathrm{a}} \pm 0.120$ & $4.50^{\mathrm{ab}} \pm 0.133$ & $4.40^{\mathrm{ab}} \pm 0.140$ & $4.03^{b} \pm 0.131$ \\
\hline Número de elementos de perianto & $19.13 \mathrm{a} \pm 0.39$ & $16.90^{\mathrm{b}} \pm 0.44$ & $16.60^{\mathrm{b}} \pm 0.499$ & $15.80^{\mathrm{b}} \pm 0.326$ \\
\hline Número de óvulos & $101.77^{\mathrm{a}} \pm 4.57$ & $94.20^{\mathrm{ac}} \pm 5.41$ & $72.73^{b} \pm 4.91$ & $75.63^{\mathrm{bc}} \pm 3.370$ \\
\hline Hercogamia & Sí $(p=0.0014)$ & No $(p=0.0808)$ & Sí $(p=0.0119)$ & No $(p=0.254)$ \\
\hline
\end{tabular}

Estudios realizados en cactáceas han detectado variación intraespecífica e intrapoblacional en las estructuras florales relacionadas a factores abióticos (Schlumpberger et al. 2009, Gutiérrez-Flores et al. 2017, Aguilar-García et al. 2018). Durante el pico de floración de M. geometrizans encontramos variación en la morfología floral entre reservas. En general, en la Reserva de Metztitlán, hubo flores de menor tamaño, con mayor número de estructuras florales. Mientras que, en la Reserva de Tehuacán-Cuicatlán se encontraron flores más grandes con menor número de estructuras florales. Estos resultados indican un posible compromiso, en las flores de $M$. geometrizans, entre tamaño floral y número de estructuras florales, lo que coincide con otros sistemas en los que se ha documentado que el aumento en la inversión a una función puede disminuir la inversión en otras (Harder \& Barrett 2006, Vasconcelos \& Proença 2015). Ambas estrategias (tamaño vs número de estructuras florales) pueden favorecer el éxito reproductivo de las plantas debido a lo atractivo que resultan para los visitadores florales, incluyendo a los polinizadores (Friedman \& Barrett 2011). Al respecto, Gutiérrez-Flores et al. (2017) sugieren que, bajo condiciones estresantes, las flores decrecen en tamaño, pero incrementan el número de sus estructuras asociadas a las funciones reproductivas. Este resultado coincide con nuestros datos, porque las flores de la Reserva de la Barranca de Metztitlán se enfrentan a mayor disturbio antrópico lo cual podría implicar mayor estrés, a diferencia de las flores de Tehuacán que son más grandes, con menos estructuras florales y menor estrés antrópico.
Con respecto a la hercogamia, las flores de M. geometrizans también presentaron características que se relacionan con el tipo de vegetación en el que se encuentran. En las selvas bajas (2TSB y 4MSB) predominó el morfo Pin y en los matorrales (1MMX y 3TMX) el morfo Thrum. En el caso del morfo Thrum se ha reportado que puede estar favoreciendo la exportación de polen (MartínezRamos et al. 2017). Por lo anterior, sería interesante evaluar si existe una relación entre este morfo y la función masculina en $M$. geometrizans, debido a que en este trabajo se identificó mayor número de estambres en las flores con morfo Thrum. El morfo Pin, que favorece la función femenina de las flores, fue más abundante en las selvas bajas. Sin embargo, la diferencia no fue significativa, por lo anterior, sería recomendable ampliar el muestreo para confirmar este resultado

La riqueza de la comunidad de artrópodos recolectada en este trabajo fue mayor a la reportada para otras especies de cactáceas con antesis diurna (Blair \& Williamson 2008, Martínez-Adriano et al. 2015, Vázquez et al. 2016, Córdova-Acosta 2017). Sin embargo, a diferencia de $M$. geometrizans, estas especies presentan flores más grandes y menor cantidad de flores por planta. Maqueda-Díaz \& Callejas-Chavero (2018), en un estudio realizado en una localidad del estado de Hidalgo, reportaron una riqueza de artrópodos similar. En ambos trabajos, el orden Hymenoptera fue el visitador predominante en las flores del garambullo, seguido por Diptera y Coleoptera. La alta diversidad de artrópodos encontrada en este sistema puede 


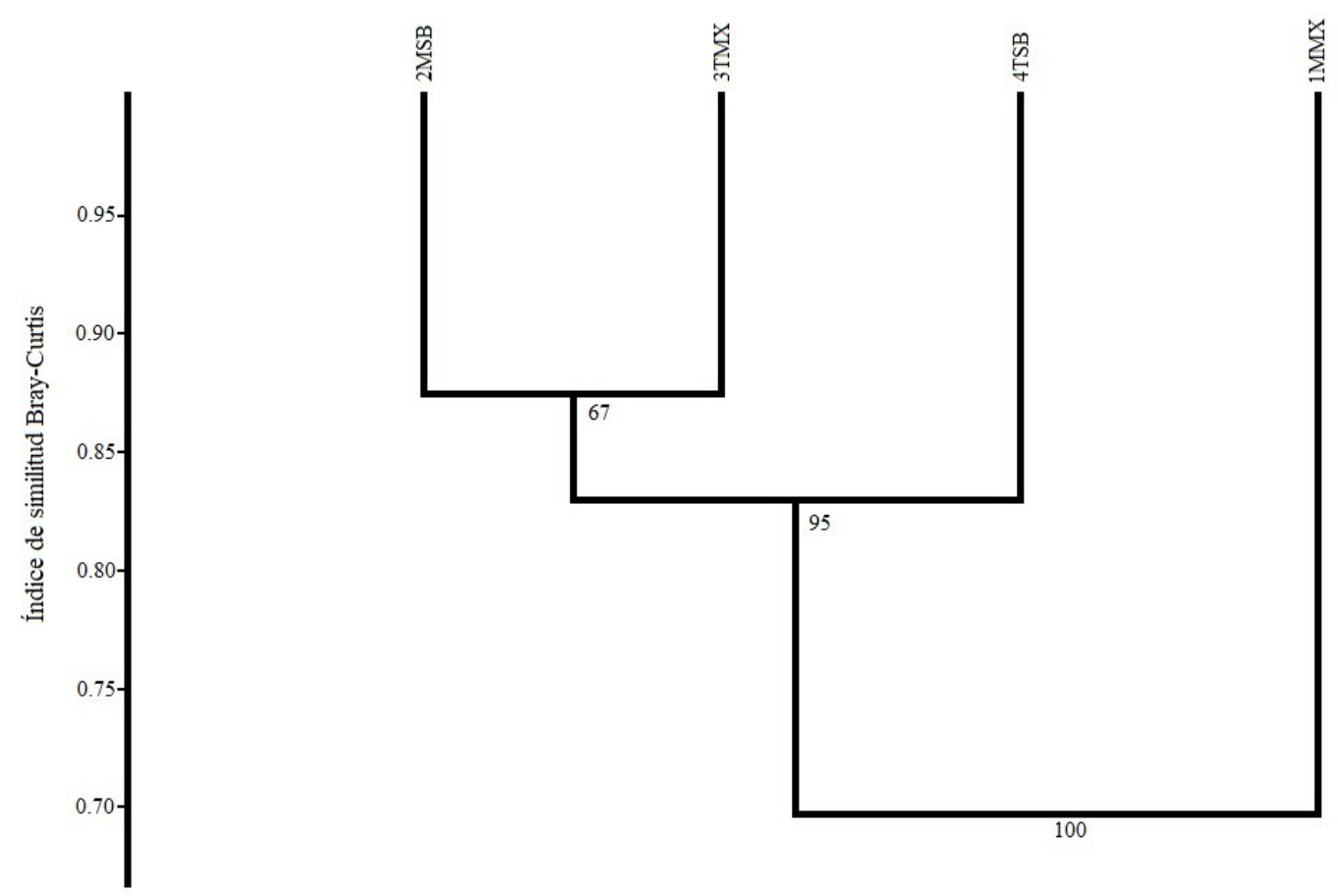

Figura 4. Dendrograma resultado de un análisis de clasificación jerárquica con el método UPGMA basado en la similitud de Bray-Curtis entre las comunidades de artrópodos visitadores de flores de M. geometrizans en los cuatro sitios de estudio (correlación cofenética de 0.85). Se indica el valor de soporte de cada rama.

deberse a que $M$. geometrizans presenta su periodo de floración en una etapa del año diferente a la mayoría de las especies, por lo que es un recurso importante para la comunidad de artrópodos (Maqueda-Díaz \& CallejasChavero 2018).

Las comunidades de artrópodos visitadores presentaron mayor abundancia en los matorrales, particularmente en el sitio 1MMX. Las flores de las selvas bajas de ambas reservas presentaron el menor número de visitadores, particularmente el sitio 4TSB. Las flores de los matorrales xerófilos en ambas reservas tuvieron la comunidad de artrópodos más compleja, con el mayor número de familias y grupos funcionales. El número de grupos funcionales fue similar al encontrado por Martínez-Adriano et al. 2015 en las flores de Astrophytum myriostigma. Una característica importante en las flores de los matorrales es la dominancia del morfo Thrum. En este morfo el polen queda más expuesto, lo cual es atractivo para los artrópodos (Hernández-Ramírez 2018). Al respecto, se ha reportado en otros sistemas que este morfo recibe un mayor número de visitadores florales (Hernández-Ramírez 2018). El sitio 4TSB fue el que tuvo menos visitadores, en este sitio se presentó una mayor abundancia de depredadores, lo que puede estar ejerciendo un control de las poblaciones del resto de artrópodos. El ID de los sitios, también puede ser un factor que esté influyendo en la comunidad de visitadores florales. Las flores del sitio más perturbado, $1 \mathrm{MMX}$, fueron las que recibieron un mayor número de visitadores florales, mientras que las del sitio 4TSB, que es de los menos perturbados, presentó menos visitadores. Este resultado contrasta con el que reportaron Juárez-Sotelo et al. (2018) en selvas bajas caducifolias, donde el sitio menos perturbado fue el que tuvo mayor diversidad de artrópodos. También, difiere con el caso de las abejas visitadoras de Opuntia heliabravoana Scheinvar 1974, que presentaron menor abundancia en sitios más perturbados (SánchezEcheverría et al. 2016). Sin embargo, se ha documentado que los cambios provocados por la perturbación antrópica generan diversos microambientes que favorecen la colonización de especies de artrópodos (Yang et al. 2015, Juárez-Sotelo et al. 2018). Sobre todo, de aquellas que presentan conducta de anidación, comportamiento social y hábitos alimenticios generalistas (Sánchez-Echeverría et al. $\underline{2016}$ ).

En conclusión, los resultados de este estudio indicaron que las flores de $M$. geometrizans no tienen mayor similitud morfológica relacionada al tipo de vegetación como lo planteado en nuestra hipótesis. Al parecer la cercanía geográfica influye más que el tipo de vegetación en la morfología floral. También, se identificó que el tamaño de 


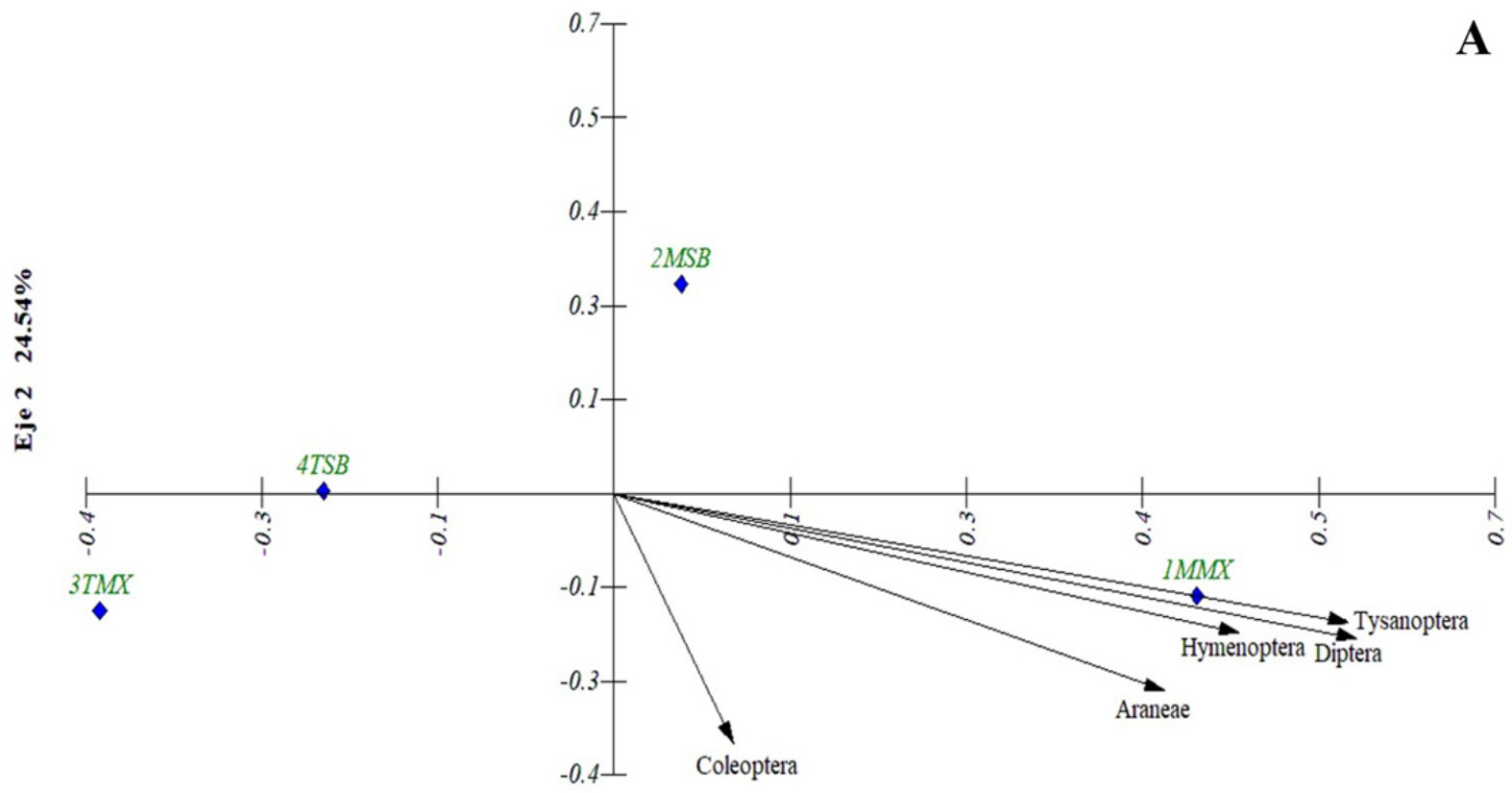

Eje $170.56 \%$

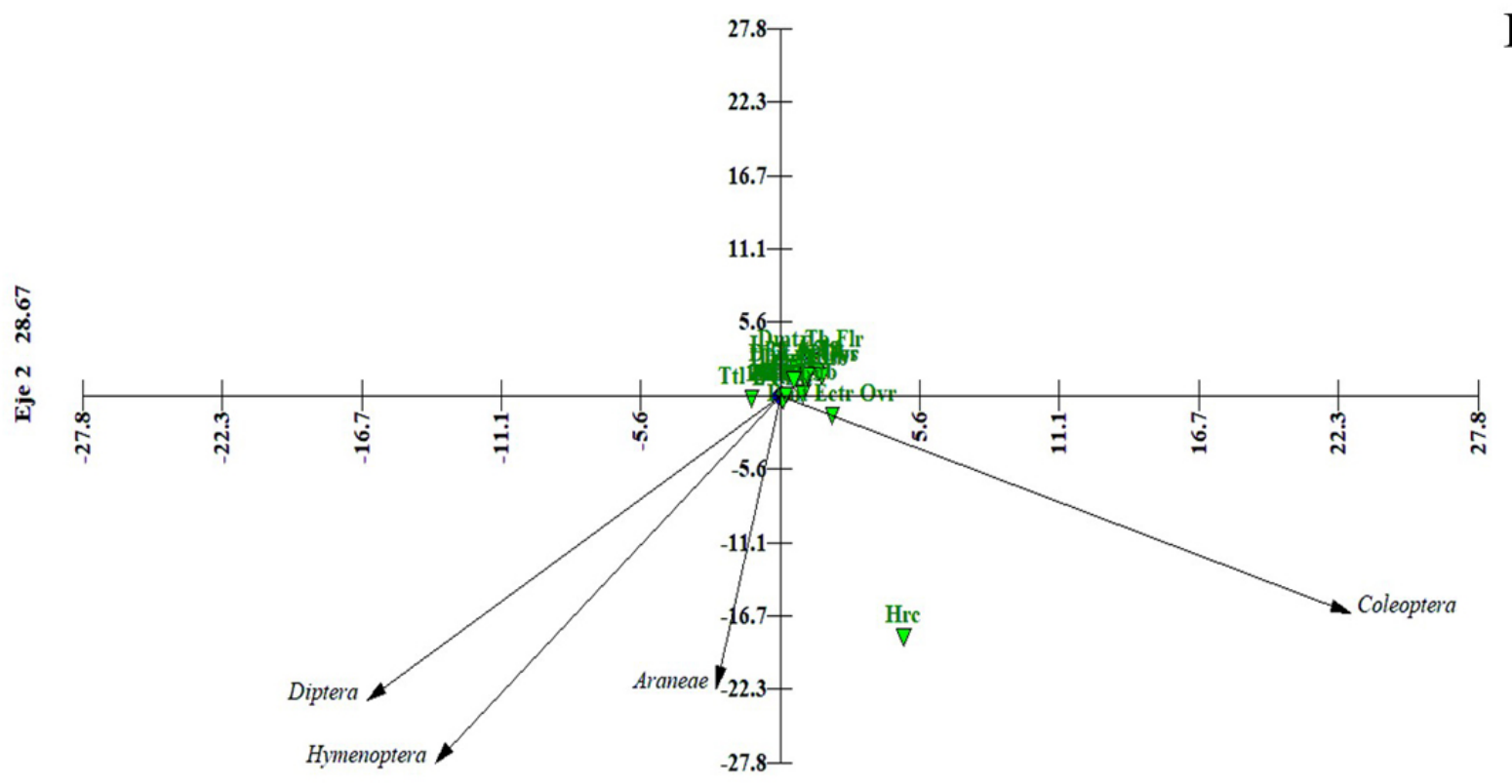

Eje $1 \quad 68.85 \%$

Figura 5. Gráfico del análisis canónico de correspondencia (ACC) que muestra la abundancia de los órdenes de artrópodos visitadores de las flores de M. geometrizans (vectores) con respecto a los sitios que se encuentran en un gradiente de disturbio (rombos azules) (A) y la abundancia de los órdenes de artrópodos (vectores) con respecto a las estructuras florales (triángulos verdes) (B). La longitud de los vectores está escalada por un factor de 2.00 (A) y 626.26 (B) para facilitar su visibilidad con respecto a la dispersión de los puntos en el espacio de ordenación. 
la flor no fue la característica más atractiva para los artrópodos visitadores florales como se esperaba, porque las flores de menor tamaño, pero con un número relativamente mayor de estructuras florales fueron las más visitadas. Además, de acuerdo con nuestra hipótesis, el sitio más perturbado presentó mayor abundancia de artrópodos y de morfoespecies de visitadores florales, quizá por la generación de microambientes que favorecen el establecimiento de algunas especies.

Un hallazgo importante en el presente trabajo, adicional a nuestras hipótesis de investigación, fue la manera en que M. geometrizans maneja un balance costo/beneficio en su morfología floral al presentar un compromiso entre el tamaño de la flor y el número de estructuras florales durante su pico de floración.

Finalmente, en este estudio se revelaron aspectos interesantes sobre la morfología floral y la comunidad de artrópodos visitadores de las flores de M. geometrizans, durante el pico de floración, en dos Reservas de la Biósfera de México. Sin embargo, consideramos que se complementaría con: 1) Un análisis de la diversidad y estructura genética en relación con las características florales. 2) Un estudio detallado sobre la biología de los visitadores florales. 3) Realizar un análisis sobre las redes de interacción del garambullo con sus visitadores y entre estos. 4) Investigar la variación intra- e inter-anual de la morfología floral y de las relaciones con sus visitadores durante todo el periodo de floración de $M$. geometrizans, para conocer completa la dinámica de este sistema.

\section{Agradecimientos}

La primera autora agradece el apoyo al Consejo Nacional para la Ciencia y Tecnología por la beca otorgada (298646). Agradecemos a las personas que nos ayudaron en campo y/o en el laboratorio para obtener los datos, A. Orozco Robles, C. Fernández Rendón, C. Rubín Sánchez, L.M. Andrade Muñiz, L. Esquibel Suárez, L.E. Soto Cortes, M.A. González Adán; al guía de la Reserva Tehuacán-Cuicatlán, P. Miranda; al editor asociado y a dos revisores anónimos que ayudaron a mejorar el escrito.

\section{Literatura citada}

Aguilar-García SA, Figueroa-Castro DM, Valverde PL, Vite F. 2018. Effect of flower orientation on the male and female traits of Myrtillocactus geometrizans (Cactaceae). Plant Biology 20: 531-536. DOI: https://doi.org/10.1111/ plb.12713

Aizen MA. 2007. Enfoques en el estudio de la reproducción sexual de las plantas en ambientes alterados: limitaciones y perspectivas. Ecología Austral 17: 7-19.
Arias S, Gamma-López S, Guzmán LU. 1997. Flora del Valle de Tehuacán-Cuicatlán. Cactaceae A.L. Juss. Fascículo 14. México, DF: Universidad Nacional Autónoma de MéxicoISBN: 968-36-3108-8.

Arias S, Guzmán U, Mandujano MC, Soto M, Golubov J. 2005. Las especies mexicanas de cactáceas en riesgo de extinción. I. Una comparación entre los listados NOM-059-ECOL-2001 (México), La lista Roja (UICN) y CITES. Cactáceas y Suculentas Mexicanas 50: 100-125

Armbruster WS. 1997. Exaptations link evolution of plantherbivore and plant-pollinator interactions: a phylogenetic inquiry. Ecology 78: 1661-1672. DOI: https://doi.org/10.1890/0012-9658(1997)078[1661:ELE $\mathrm{OPH}] 2.0 . \mathrm{CO} ; 2$

Barrett SC. 2010. Understanding plant reproductive diversity: Biological Sciences 365: 99-109. DOI: https:// doi.org/10.1098/rstb.2009.0199

Blair AW, Williamson PS. 2008. Effectiveness and importance of pollinators to the star cactus (Astrophytum asterias). The Southwestern Naturalist 53: 423-430. DOI: https://doi.org/10.1894/JB-04.1

Borror DJ, Triplehorn CA, Johnson NF. 1989. An introduction to the study of insects. Philadelphia, USA: Saunders College Publishing. ISBN: 0030253977

Bravo-Hollis H, Sánchez-Mejorada R. 1978. Datos acerca de la ecología de las cactáceas y de su distribución en los tipos de vegetación en México. In: Bravo-Hollis $\mathrm{H}$, Sánchez-Mejorada R, eds. Las Cactáceas de México. Vol. I. México: Universidad Nacional Autónoma de México. pp. 84-123. ISBN: 9683617581, 9789683617583.

Bravo-Hollis H, Sánchez-Mejorada R. 1991. Tribu V Cacteae. In: Bravo-Hollis H, Sánchez-Mejorada R, eds. Las Cactáceas de México. Vol. II. México: Universidad Nacional Autónoma de México. pp. 102-122. ISBN: 968361759X, 9789683617590.

Cardel Y, Rico-Gray V, García-Franco JG, Thien LB. 1997. Ecological status of Beaucarnea gracilis, an endemic species of the semiarid Tehuacán Valley, México: Conservation Biology 11: 367-374. DOI: https://doi.org/ 10.1046/j.1523-1739.1997.95322.x

Casas A, Caballero J, Valiente-Banuet A. 1999. Use, management and domestication of columnar cacti in south-central Mexico: A historical perspective. Journal of Ethnobiology 19: 71-95.

CONANP [Comisión Nacional de Áreas Naturales Protegidas]. 2003. Programa de Manejo de la Reserva de la Biosfera Barranca de Metztitlán. Comisión Nacional de Áreas Naturales Protegidas, México. Secretaría de Medio Ambiente y Recursos Naturales. ISBN: 968-817-592-7 
Córdova-Acosta E, Zavala-Hurtado JA, Golubov J, Casas A. 2017. Reproductive biology of Ferocactus recurvus (Mill.) Borg subsp. recurvus (Cactaceae) in the Tehuacán-Cuicatlán Valley, Mexico. Plant Biology 19: 798-805. DOI: https://doi.org/10.1111/plb.12585

Friedman J, Barrett SC. 2011. The evolution of ovule number and flower size in wind-pollinated plants. The American Naturalist 177: 246-257. DOI: https://doi.org/ $\underline{10.1086 / 657954}$

Ganders FR. 1979. The biology of heterostyly. New Zealand Journal of Botany 17: 607-635. DOI: https:// doi.org/10.1080/0028825X.1979.10432574

Geber MA, Moeller DA. 2006. Pollinator responses to plant communities and implications for reproductive character evolution. In: Harder LD, Barrett SC, eds. 2006. Ecology and Evolution of Flowers. United States, New York: Oxford University Press. pp. 2-42. ISBN: 0-19-857085-6.

Gutiérrez-Flores C, Cota-Sánchez JH, León-de la Luz JL, García-De León FJ. 2017. Disparity in floral traits and breeding systems in the iconic columnar cactus Pachycereus pringlei (Cactaceae). Flora 235: 18-28. DOI: https://doi.org/10.1016/j.flora.2017.08.007

Hammer O, Harper DAT, Ryan PD. 2001. PAST: Paleontological statistics software package for education and data analysis. Paleontologia Electronica 4: 1-9.

Harder LD, Barrett SC. 2006. David G. Lloyd and the evolution of floral biology: from natural history to strategic analysis. En: Harder LD, Barrett SC, eds. 2006. Ecology and evolution of flowers. Oxford University Press on Demand, pp. 2-42. ISBN: 0-19-857085-6.

Hernández-Ramírez AM. 2018. Buscando al polinizador eficiente: variación temporal en el gremio de visitantes florales y carga polínica estigmática en la especie distílica Palicourea padifolia (Rubiaceae). Revista Mexicana de Biodiversidad, 89: 412-420. DOI: https:// doi.org/10.22201/ib.20078706e.2018.2.2369

Juárez-Sotelo LE, Olalde-Estrada I, Castellanos-Vargas I, Cano-Santana Z. 2018 Comunidades de artrópodos terrestres en sitios perturbados de selva baja caducifolia del ejido San José Tilapa, Coxcatlán, Puebla, México. Entomología Mexicana 5: 294-300.

Kovach WL. 2004. MVSP-A MultiVariate Statistical Package for Windows, Version 3.13. Pentraeth, Wales: Kovach Computing Services.

Krishnadas M, Bagchi R, Sridhara S, Comita LS. 2018. Weaker plant-enemy interactions decrease tree seedling diversity with edge-effects in a fragmented tropical forest. Nature Communications 9: 1-7. DOI: https:// doi.org/10.1038/s41467-018-06997-2

Luna de la Torre P, Castro-Leal L, Contreras-Cerón R, Castillo-Meza AL. 2016. Actividad de formícidos en Ferocactus latispinus (Cactaceae) en una zona semiárida del centro de México. Entomología Mexicana 3: 530-536.

Mandujano MC, Carrillo-Ángeles IG, Martínez-Peralta C, Golubov J. 2010. Reproductive biology of Cactaceae. In: Ramawat KG, ed. Desert Plants Biology and Biotechnology. Berlin, Germany: Springer, pp. 197-230. ISBN: 978-3-642-02550-1.

Maqueda-Díaz E, Callejas-Chavero A. 2018. Riqueza y composición de artrópodos asociados a las flores de Myrtillocactus geometrizans (Mart. ex Pfeiff.) Console, 1897 en Huichapan, Hidalgo. Entomología Mexicana 5: 267-273.

Martínez-Adriano CA, Romero-Méndez U, Flores J, Jurado E, Estrada-Castillón E. 2015. Floral visitors of Astrophytum myriostigma in La Sierra El Sarnoso, Durango, Mexico. The Southwestern Naturalist 60: 158-165. DOI: https://doi.org/10.1894/FMO-12.1

Martínez-Hernández DG, Callejas-Chavero A. 2015. Efecto mutualista de Liometopum apiculatum (Hymenoptera: Formicidae) sobre la tasa de parasitoidismo en Toumeyella martinezi (Hemiptera: Coccidae) asociados a Myrtillocactus geometrizans (Cactaceae). In: CastañoMeneses G, Vásquez-Bolaños M, Navarrete-Heredia JL, Quiroz-Rocha GA, Alcalá-Martínez I, eds. Avances de Formicidae de México. Guadalajara, Jalisco, México: Astra Editores. pp. 109-120. ISBN: 978-607-9450-01-4.

Martínez-Ramos LM, Mejía-Rojas M, Rojas-Aréchiga M, Mandujano MC. 2017. La hercogamia como indicador del sistema reproductivo de Thelocactus leucacanthus spp. schmollii. Cactáceas y Suculentas Mexicanas 62: 13-22.

Martorell C, Peters EM. 2005. The measurement of chronic disturbance and its effects on the threatened cactus Mammillaria pectinifera. Biological Conservation 124: 199-207.

Martorell C, Peters EM. 2009. Disturbance-response analysis: a method for rapid assessment of the threat to species in disturbed areas. Conservation Biology 23: 377-387. DOI: https://doi.org/10.1111/j.1523-1739.2008. 01134.x

Moreno CE, Sánchez-Rojas G, Verdú JR, Numa C, MarcosGarcía MA, Martínez-Falcón AP, Galante E, Halffter G. 2007. Biodiversidad en ambientes agropecuarios semiáridos en la Reserva de la Biósfera Barranca de Metztitlán, México. Hacia una Cultura de la Conservación de la Diversidad Biológica 6: 97-107.

Morinière J. Hendrich L. Balke M. Beermann AJ. König T. Hess M, Hausmann, A. 2017. A DNA barcode library for Germany' s mayflies, stoneflies and caddisflies (Ephemeroptera, Plecoptera and Trichoptera). Molecular Ecology Resources 17: 1293-1307. DOI: https://doi.org/ $\underline{10.1111 / 1755-0998.12683}$ 
Morón MA, Terrón RA. 1988. Entomología Práctica: una guía para el estudio de los insectos con importancia agropecuaria, médica, forestal y ecológica de México. México DF: Instituto de Ecología, A.C. ISBN: 9687213086, 9789687213088

Moya-Raygoza G, Kathirithamby J, Larsen KJ. 2004. Dry season parasitoids of adult corn leafhoppers (Hemiptera: Cicadellidae) on irrigated maize in Mexico. The Canadian Entomologist 136: 119-127. DOI: https:// doi.org/10.4039/n03-038

Navarrete-Jiménez A, Herrera-Fuentes MC, Bazán-Morales A, Zavala-Hurtado JA, Campos-Serrano J. 2018. Listado preliminar del orden Araneae en el Jardín Botánico de Zapotitlán, Puebla. Entomología Mexicana 5: 576-579.

Norusis MJ. 2011. IBM SPSS Statistics 19 Statistical Procedures Companion. Vol. 496. New Jersey, USA: Education Upper Saddle River. Prentice Hall Pearson.

Páez-Gerardo LE, Aguilar-Rodríguez SA, Terrazas T, Huidobro-Salas ME, Aguirre-León EA. 2005. Cambios anatómicos en la corteza de Parkinsonia praecox (Ruiz et Pavón) Hawkins causados por la epífita Tillandsia recurvata L. (Bromeliaceae). Boletín de la Sociedad Botánica de México 77: 59-64. DOI: https://doi.org/ $10.17129 /$ botsci. 1713

Rasband W. 1997. Software ImageJ 1.50i. USA: National Institutes of Health,

Ríos-Casanova L, Valiente-Banuet A, Rico-Gray V. 2004. Las hormigas del Valle de Tehuacán (Hymenoptera: Formicidae): una comparación con otras zonas áridas de México. Acta Zoológica Mexicana 20: 37-54.

Rosas-García EM. 2010. Efecto de la orientación preferencial sobre las estructuras reproductivas $y$ vegetativas en Myrtillocactus geometrizans. MSc. Thesis. Universidad Autónoma Metropolitana.

Rzedowski J. 2006. Vegetación de México. 1ra. Edición digital, México: Comisión Nacional para el Conocimiento y Uso de la Biodiversidad.

Sánchez-Echeverría K, Castellanos I, Mendoza-Cuenca L. 2016. Abejas visitantes florales de Opuntia heliabravoana en un gradiente de urbanización. Biológicas 18: 27-34.

Schlumpberger BO, Cocucci AA, Moré M, Sérsic AN, Raguso RA. 2009. Extreme variation in floral characters and its consequences for pollinator attraction among populations of an Andean cactus. Annals of Botany 103: 1489-1500. DOI: https://doi.org/10.1093/aob/mcp075

SEMARNAP [Secretaría de Medio Ambiente, Recursos Naturales y Pesca]. 1999. Reserva de la Biósfera Barranca de Metztitlán, Hidalgo. Documento técnico justificativo para la creación de la Reserva de la Biósfera
Barranca de Metztitlán, Hgo. Pachuca, Hidalgo, México: SEMARNAP.

Sneath PHA, Sokal RR. 1973. Numerical taxonomy. The principles and practice of numerical classification. United States, San Francisco: Freeman. ISBN: 0716706970.

Stone MJ, Catterall CP, Stork NE. 2018. Edge effects and beta diversity in ground and canopy beetle communities of fragmented subtropical forest. Plos One 13: 3. DOI: https://doi.org/10.1371/journal.pone.0193369

Strauss SY. 2012. Leaf-Beetle community structure in an Amazonian rainforest canopy. In: Jolivet P, Petitpierre E, Hsiao TH, eds. Biology of Chrysomelidae. Dordrecht, The Netherlands: Springer Science and Business Media. pp. 73. e-ISBN 978-94-009-3105-3; DOI: https://doi.org/ 10.1007/978-94-009-3105-3_5

Strauss SY, Whittall JB. 2006. Non-pollinator agents of selection on floral traits. In: Harder, LD, Barrett SC, eds. Ecology and Evolution of Flowers. United States, New York: Oxford University Press pp. 120-138. ISBN: 0-19-857085-6.

Terrón SR, Urbán CG, Rodríguez NS. 1998. Ordenes de insectos con importancia agrícola: guía diagnostica y morfología general. México: Universidad Autónoma Metropolitana.

Toft CA, Kimsey, LS. 1982. Habitat and behavior of selected Apiocera and Rhaphiomidas (Diptera, Apioceridae), and descriptions of immature stages of $A$. hispida. Journal of the Kansas Entomological Society 55: 177-186.

Triplehorn CA, Johnson NF. 2005. Borror and DeLongs Introduction to the Study of Insects. United States, Pacific Grove: Brooks/Cole Thomson Learning. ISBN: 0-03-096835-6.

Valverde PL, Vite F, Pérez-Hernández MA. Zavala-Hurtado JA. 2007. Stem tilting, pseudocephalium orientation, and stem allometry in Cephalocereus columna-trajani along a short latitudinal gradient. Plant Ecology 188: 17-27. DOI: https://doi.org/10.1007/s11258-006-9144-1

Vasconcelos TN, Proença CE. 2015. Floral cost vs. floral display: insights from the megadiverse Myrtales suggest that energetically expensive floral parts are less phylogenetically constrained. American Journal of Botany 102: 900-909. DOI: https://doi.org/10.3732/ ajb.1400509

Vázquez E, Sánchez E, Aguilar M. 2016. Biología floral de Echinocereus schmollii (Weing.) N.P. Taylor, especie endémica de Cadereyta de Montes, Querétaro, México. Cactáceas y Suculentas Mexicanas 61: 12-28. 
Islas-Barrios et al. / Botanical Sciences 99(1): 28-42. 2021

Woźniak NJ, Sicard A. 2018. Evolvability of flower geometry: convergence in pollinator-driven morphological evolution of flowers. Seminars in Cell \& Developmental Biology 79: 3-15. DOI: https://doi.org/ 10.1016/j.semcdb.2017.09.028

\section{Editor de sección: Joel Flores}

Contribución de los autores: YIB concibió la idea, diseñó el estudio, realizó el trabajo de campo, analizó los datos y escribió el manuscrito; ASD concibió la idea, consiguió financiamiento, diseñó el estudio, participó en el análisis de los datos y en la escritura del manuscrito; JAZH participó en el diseño del estudio, en el análisis de los datos y en la escritura del manuscrito; POA participó en el diseño del estudio, trabajo de campo, en el análisis estadístico y en la revisión del manuscrito; ACC participó en el desarrollo de la idea y diseño del estudio. ACR participó en el desarrollo de la idea y diseño del estudio.
Yang Z, Liu X, Zhou M, Ai D, Wang G, Wang Y, Chu C, Lundholm JT. 2015. The effect of environmental heterogeneity on species richness depends on community position along the environmental gradient. Scientific Reports 5: $15723 . \quad$ DOI: https://doi.org/10.1038/ $\underline{\text { srep } 15723}$ 
Apéndice 1. Artrópodos visitadores florales de M. geometrizans (clasificación taxonómica, grupo funcional y número de individuos) registrados en cada uno de los sitios (1MMX, Matorral Xerófilo de Metztitlán; 2MSB, Selva Baja de Metztitlán; 3TMX, Matorral Xerófilo de Tehuacán y 4TSB, Selva Baja de Tehuacán). Los grupos funcionales se identificaron de la siguiente manera: Polinizador (Plnzdr); Nectarívoro (Nctrv); Parásito de artrópodos (Prst artr); Depredador (Dprd); Herbívoro (Hrbv); Robador de néctar (Rbd nctr); Depredador, Nectarívoro (Dprd, Nctrv); Depredador, Parásito de artrópodos (Dprd, Prst artr); Depredador, Polinizador (Dprd, Plnzdr).

\begin{tabular}{|c|c|c|c|c|c|}
\hline Sitio & Orden & Familia & Morfoespecie & Grupo funcional & Total \\
\hline \multirow[t]{20}{*}{ 1MMX } & Araneae & Salticidae & Msp. 62., Msp. 59, Msp. 60 & Dprd & 3 \\
\hline & \multirow[t]{2}{*}{ Coleoptera } & Scarabaeidae & Melolonthinae sp. 72 & Hrbv & 1 \\
\hline & & Chrysomelidae & Msp. 2, Msp. 70, Msp. 71 & Hrbv & 7 \\
\hline & \multirow[t]{2}{*}{ Diptera } & Pipunculidae & Msp. 37, Msp.38 & Prst artr & 6 \\
\hline & & Syrphidae & Msp. 44, Msp. 45, Msp. 57, Msp. 68, Msp. 73, Msp. 36, Msp. 65 & Plnzdr & 17 \\
\hline & \multirow[t]{14}{*}{ Hymenoptera } & Anthophoridae & Msp. 50 & & 3 \\
\hline & & Apidae & Apis sp. 1 & Plnzdr & 25 \\
\hline & & & Msp. 40, Msp. 41, Msp. 48, Msp. 51, Msp. 69, Msp. 99 & & 15 \\
\hline & & & Msp. 14 & Rbd nctr & 1 \\
\hline & & \multirow[t]{5}{*}{ Formicidae } & Camponotus sp. 32 & Netrv & 1 \\
\hline & & & Brachymyrmex sp. 33 & & 3 \\
\hline & & & Brachymyrmex sp. 76 & & \\
\hline & & & Crematogaster sp. 30 & Dprd, Netrv & 1 \\
\hline & & & Pseudomyrmex sp. 4, P. sp. 66, P. sp. 74 & & 5 \\
\hline & & Halictidae & Msp. 5, Msp. 6, Msp. 16, Msp. 39, Msp. 55, Msp. 46 & Plnzdr & 17 \\
\hline & & Ichneumonidae & Msp. 47 & Prst artr & 1 \\
\hline & & Sphecidae & Msp. 53 & Dprd, Prst artr & 2 \\
\hline & & Vespidae & Msp. 56, Msp. 67 & Plnzdr & 16 \\
\hline & & & Msp. 102 & Rbd nctr & 1 \\
\hline & Thysanoptera & Thripidae & Msp. 58 & Nctrv & 1 \\
\hline \multirow[t]{13}{*}{ 2MSB } & \multirow[t]{3}{*}{ Diptera } & Dolochopodidae & Msp. 43 & Dprd & 1 \\
\hline & & Pipunculidae & Msp. 38 & Prst artr & 2 \\
\hline & & Syrphidae & Msp. 22, Msp. 23 & Plnzdr & 3 \\
\hline & \multirow[t]{10}{*}{ Hymenoptera } & Apidae & Apis sp. 1 & Plnzdr & 19 \\
\hline & & & Msp. 41, Msp. 79 & & 13 \\
\hline & & Chalcididae & Msp. 54 & Prst artr & 1 \\
\hline & & Formicidae & Msp. 25, M sp. 93 & Netrv & 2 \\
\hline & & & Camponotus sp. 32 & Netrv & 3 \\
\hline & & & Crematogaster sp. 30 & Dprd, Nctrv & 1 \\
\hline & & & Pseudomyrmex sp. 42 & Dprd, Nctrv & 3 \\
\hline & & Halictidae & Msp. 5, Msp. 16, Msp. 77 & Plnzdr & 7 \\
\hline & & Ichneumonidae & Msp. 78 & Prst artr & 1 \\
\hline & & Vespidae & Msp. 64 & Rbd nctr & 7 \\
\hline
\end{tabular}


Islas-Barrios et al. / Botanical Sciences 99(1): 28-42. 2021

\begin{tabular}{|c|c|c|c|c|c|}
\hline Sitio & Orden & Familia & Morfoespecie & Grupo funcional & Total \\
\hline \multirow[t]{16}{*}{ 3TMX } & Araneae & Thomisidae & Msp. 71 & Dprd & 1 \\
\hline & Coleoptera & Chysomelidae & Msp. 2, Msp. 95, Msp. 12, Msp. 20, Msp. 21 & Hrbv & 7 \\
\hline & Diptera & Apioceridae & Msp. 18 & & 1 \\
\hline & & Muscidae & Msp. 80, Msp. 8, Msp. 9 & Dprd & 3 \\
\hline & & Pipunculidae & Msp. 37, Msp. 17, Msp. 19 & Prst artr & 3 \\
\hline & Hymenoptera & Apidae & Msp. 81 & Plnzdr & 1 \\
\hline & & Apidae & Apis sp. 1 & Plnzdr & 15 \\
\hline & & Apidae & Msp. 7, Msp. 81 & Plnzdr & 4 \\
\hline & & Formicidae & Camponotus sp. 32, C. sp. 94, C. sp. 97 & Netrv & 9 \\
\hline & & & Msp. 25 & Netrv & 2 \\
\hline & & & Msp. 15, Msp. 24, Msp. 29 & Dprd, Nctrv & 7 \\
\hline & & & Pseudomyrmex sp. 27 & Dprd, Nctrv & 2 \\
\hline & & & $P$. sp. $26, P$. sp. 31 & Dprd, Nctrv & 4 \\
\hline & & & Msp. 28, Msp. 98 & Nctrv & 2 \\
\hline & & Halictidae & Msp. 5, Msp. 6 & Plnzdr & 6 \\
\hline & & Vespidae & Msp. 34, Msp. 35, Msp. 96, Msp. 83 & Dprd, Plnzdr & 14 \\
\hline \multirow[t]{12}{*}{ 4TSB } & Araneae & Salticidae & Msp.88 & Dprd & 1 \\
\hline & Coleoptera & Chysomelidae & Msp.2, Msp.92 & Hrbv & 7 \\
\hline & Diptera & Pipunculidae & Msp.37, Msp.4 & Prst artr & 2 \\
\hline & & Muscidae & Msp.87, Msp.8, Msp.9, Msp.11 & Dprd & 4 \\
\hline & Hymenoptera & Apidae & Msp.7 & Plnzdr & 2 \\
\hline & & & Apis sp.1 & Plnzdr & 7 \\
\hline & & & Msp. 89, Msp. 82, Msp. 85, Msp. 100 & Plnzdr & 15 \\
\hline & & Formicidae & Msp. 25, Msp. 101 & Netrv & 5 \\
\hline & & & Msp. 75, Msp. 84 & Dprd, Nctrv & 3 \\
\hline & & & Pseudomyrmex sp. 10, P. sp. 86 & Dprd, Nctrv & 6 \\
\hline & & Halictidae & Msp. 5 & Plnzdr & 2 \\
\hline & & Vespidae & Msp. 34, Msp. 35, Msp. 90, Msp. 91, Msp. 89 & Dprd, Plnzdr & 6 \\
\hline
\end{tabular}

